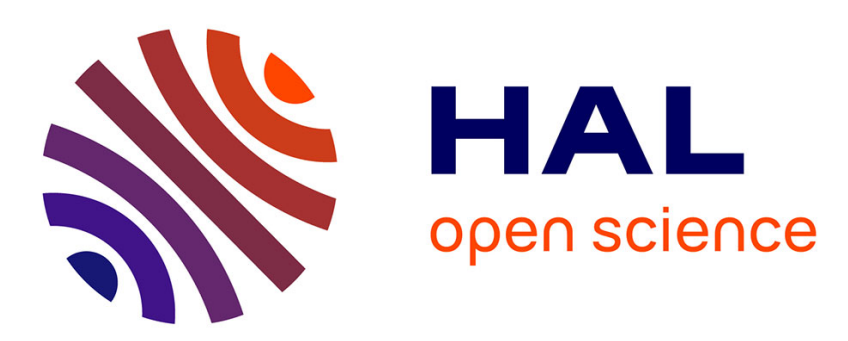

\title{
Nonadiabatic Dynamics of Charge-Transfer States Using the Anthracene-Tetracyanoethylene Complex as Prototype
}

Farhan Siddique, Mario Barbatti, Zhonghua Cui, Hans Lischka, Adélia Aquino

\section{- To cite this version:}

Farhan Siddique, Mario Barbatti, Zhonghua Cui, Hans Lischka, Adélia Aquino. Nonadiabatic Dynamics of Charge-Transfer States Using the Anthracene-Tetracyanoethylene Complex as Prototype. Journal of Physical Chemistry A, 2020, 124 (17), pp.3347-3357. 10.1021/acs.jpca.0c01900 . hal02612347

\section{HAL Id: hal-02612347 \\ https://hal-amu.archives-ouvertes.fr/hal-02612347}

Submitted on 19 May 2020

HAL is a multi-disciplinary open access archive for the deposit and dissemination of scientific research documents, whether they are published or not. The documents may come from teaching and research institutions in France or abroad, or from public or private research centers.
L'archive ouverte pluridisciplinaire HAL, est destinée au dépôt et à la diffusion de documents scientifiques de niveau recherche, publiés ou non, émanant des établissements d'enseignement et de recherche français ou étrangers, des laboratoires publics ou privés. 


\section{Nonadiabatic Dynamics of Charge-Transfer States Using the Anthracene- Tetracyanoethylene Complex as Prototype}

Farhan Siddique, ${ }^{\mathrm{a}}$ Mario Barbatti, ${ }^{\mathrm{b}}$ Zhonghua Cui, ${ }^{\mathrm{c}, \mathrm{d}}$ Hans Lischka, ${ }^{\mathrm{a}, \mathrm{e} *}$ Adelia J. A. Aquino ${ }^{\mathrm{a}, \mathrm{f} *}$

${ }^{a}$ School of Pharmaceutical Science and Technology, Tianjin University, Tianjin 300072, China

${ }^{\mathrm{b}}$ Aix Marseille University, CNRS, ICR, Marseille, France

'Institute of Atomic and Molecular Physics, Jilin University, Changchun, China

${ }^{\mathrm{d} B e i j i n g ~ N a t i o n a l ~ L a b o r a t o r y ~ f o r ~ M o l e c u l a r ~ S c i e n c e s, ~ B e i j i n g, ~ C h i n a ~}$

${ }^{\mathrm{e}}$ Department of Chemistry and Biochemistry, Texas Tech University, Lubbock, Texas 79409-1061, USA

${ }_{\mathrm{f}}^{\mathrm{f}}$ Department of Mechanical Engineering, Texas Tech University, Lubbock, TX, 79409, USA 


\section{Abstract}

Surface hopping QM/MM dynamics simulations have been performed for the tetracyanoethylene (TCNE)/anthracene complex to investigate the evolution of charge-transfer states after excitation into a locally excited (LE) state of anthracene. The scaled opposite-spin (SOS) second-order algebraic diagrammatic construction (SOS-ADC(2)) has been used to achieve a balanced description of LE and charge transfer (CT) states. The calculations have been performed for two media, the gas phase and water (described by molecular mechanics, MM). The two dynamics variants show strongly different behavior. Even though in both cases, the conversion from the LE state to lower-lying CT states occurs with $100 \mathrm{fs}$, in the gas phase, the system remains in the excited states for longer than 2 ps while in water it returns to the ground state within 0.5 ps. Moreover, while in the gas phase the original neutral equilibrium structure should be recovered, in water, the ion-pair CT state is strongly stabilized, creating a new competing ground-state isomer. Thus, we predict that the ground state of the complex in water should be composed of two species, the original neutral state and an ion-pair state. The existence of an ion-pair ground state in strongly polar environments opens interesting possibilities for the design of efficient charge-separating organic donor-acceptor interfaces. 


\section{Introduction}

The theory and understanding of intermolecular charge transfer (CT) processes ${ }^{1,2}$ is a fascinating topic because of the wide appearance and significance of this phenomenon. CT complexes are attractive candidates for constructing materials with outstanding electrical and optical properties ${ }^{3-}$ ${ }^{6}$. Mixed stacked donor (D)/acceptor (A) CT systems have been extensively investigated in recent research due to their promising features as semi-conductuctors ${ }^{7,8}$ to be used, for example, as biosensors in medicine ${ }^{9-11}$ or as for the constructions of ferroelectric crystals ${ }^{12,13}$. The formation of the CT states generated from aggregates of $\pi$-conjugated donor polymers and electron acceptors (mostly fullerenes) is also a major step in organic photovoltaic systems, ${ }^{14-16}$ in which light absorption by the donor and exciton formation in the $\pi$-conjugated chain ${ }^{17}$ act as primary processes. Theoretical investigations provide useful access to the description of the CT states, their energetic relation to the locally excited excitonic states, and their electronic response to changes in the environment ${ }^{18-23}$.

Tetracyanoethylene (TCNE) is an interesting acceptor, which forms prototypical complexes with aromatic hydrocarbons such as benzene, naphthalene, or anthracene as donors. ${ }^{24-26}$ It has been found $^{27}$ that the $\mathrm{CT}$ in the ground state is relatively small and amounts to about $0.2 e$, but pronounced $\mathrm{CT}$ bands are observed on electronic excitation, and the $\mathrm{CT}$ states usually form the lowest excited $\operatorname{states}^{27}$. The theoretical description frequently faces severe problems since the widely used time-dependent density functional theory (TD-DFT) shows, for many functionals, an artificial over-stabilization of the CT states. Therefore, range-separated functionals ${ }^{28-32}$ have been developed to circumvent this problem. On the other hand, ab initio, wave function-based methods do not show this bias but are usually computationally more expensive.

A method that has been shown as a good compromise in terms of accuracy and computational efficiency is the second-order algebraic diagrammatic construction $(\mathrm{ADC}(2))_{\text {method }}{ }^{33}{ }^{34}$, which has been successfully used in many calculations of excited states of $\pi$-conjugated systems $s^{35-38}$. For more information on the applicability of the $\mathrm{ADC}(2)$ method, also see the overviews given in Refs 39, 40. A crucial role for the success of this method can be found in combination with the resolution-of-the-identity (RI) approach ${ }^{41,42}$, which lows efficient handling of the two-electron integrals. Using empirical scaled opposite-spin (SOS) ${ }^{43,44}$ corrections, the interactions of TCNE and the aromatic compounds benzene, naphthalene, and anthracene have been investigated at SOS 
second-order Møller-Plesset perturbation theory ${ }^{45}$ (SOS-MP2) and SOS-ADC(2) level ${ }^{27}$. Computed CT bands gave good agreement with experimental measurements, and solvent effects could be well-represented at the level of the conductor-like screening model (COSMO) ${ }^{46,47}$ using dichloromethane as solvent. An interesting feature was observed in these investigations when calculating vertical emission processes from the $S_{1}$ state. Significant stabilization of the $S_{1}$ state by the solvent was found for all three complexes, which led, especially for the anthracene case, to a zero-energy gap between excited and ground states. This circumstance opens the possibility for fluorescence quenching due to ultrafast radiationless deactivation to the ground state.

The purpose of the present work is to perform a photodynamical characterization of the radiationless deactivation of the TCNE/anthracene complex to the ground state. The motivation for the choice of the TCNE/anthracene complex (Figure 1) lies in the fact that it offers the possibility of a prototypical study of the sequence of events starting from a bright, locally excited $\pi-\pi^{*}$ state followed by the internal conversion to lower-lying CT states, and final decay to the ground state. The reason for the choice of the anthracene complex was also given by the observation in our previous static calculations on the series of TCNE complexes with benzene, naphthalene and anthracene ${ }^{27}$ that with increasing size of these polycyclic aromatic hydrocarbons the fluorescence energy systematically decreased, and that for the anthracene complex this energy was practically zero. This fact indicated rapid internal conversion to the ground state. This strong stabilization of the CT state was closely connected to solvent effects and did not occur in gas phase. According to our previous calculations, this should have happened for dichloromethane. To assure the internal conversion process in the present, time-consuming on-the-fly dynamics, we decided to use as solvent water with an even larger dielectric constant.

The photodynamical studies have been performed through decoherence-corrected fewest switches surface hopping dynamics ${ }^{48}$ for two different environments, the isolated complex and aqueous solution. The SOS-ADC(2) method has been used as a reliable method to describe CT complexes, as discussed above. Solvent effects have been included explicitly at the atomistic level by means of the quantum mechanical/molecular dynamics (QM/MM) approach ${ }^{49-52}$. These nonadiabatic dynamics simulations are well suited to provide insight into the details of the deactivation processes of CT states and about the role, polar solvation is playing. 
a)

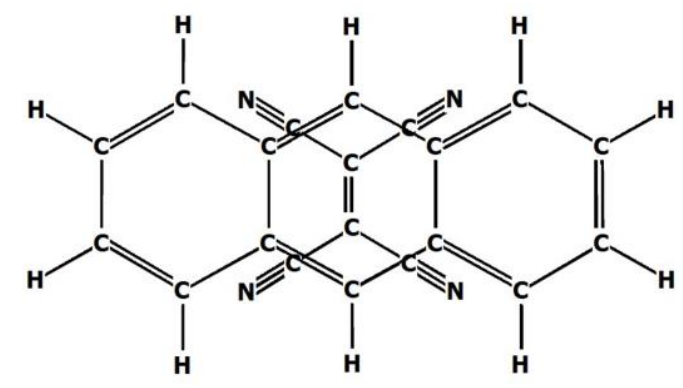

b)
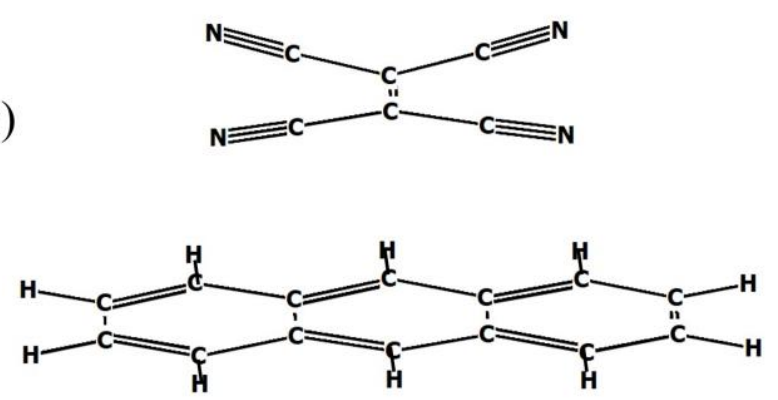

Figure 1: Top (a) and side (b) views of the anthracene-TCNE complex in the ground state.

\section{Computational Details}

Optimization of ground-state geometries was performed with the SOS-MP2 method, and excited states were calculated with the SOS-ADC(2) method using the RI approach and the SV(P) basis set. ${ }^{40}$ Solvation effects for water were treated for vertical excitations using the conductorlike screening model (COSMO $)^{46,47}$ based on a continuum approach. The values of $\varepsilon=78.39^{53}$ for the relative dielectric constant and $n=1.33^{54}$ for the refractive index of water were used. Ground state optimizations using COSMO were performed with the PTE scheme ${ }^{55}$. For the nonadiabatic dynamics in water and the vibrational broadening of the UV spectra (see below), an additive QM/MM scheme ${ }^{56}$ with water as the solvent has been applied as described in Refs ${ }^{52,57}$. In this approach, the total region $S$ of the solute/solvent system is divided into an inner region $I$ for the QM part and an outer MM region $O$ for the solvent. The total energy of the entire system $S$ is written as

$$
E_{\mathrm{QM} / \mathrm{MM}}(S)=E_{\mathrm{QM}}(I)+E^{\mathrm{el}}(I, O)+E_{\mathrm{MM}}^{\mathrm{vdW}}(I, O)+E_{\mathrm{MM}}^{\mathrm{b}+\mathrm{vdW}}(O)+E_{\mathrm{MM}}^{\mathrm{el}}(O),
$$

where the superscripts denote electronic (el), van der Waals (vdW), and bonding (b). 
The QM part consisted of the TCNE/anthracene complex. It was included in a spherical cluster of 1000 water molecules, and the initial packing was performed using the PACKMOL program ${ }^{58}$. To keep the gross density during the dynamics constant, the entire cluster was constrained into a spherical boundary for the entire time of the simulation. The radius of the sphere was $19.3 \AA$ to obtain a water density of $0.997 \mathrm{~g} / \mathrm{L}^{59}$. Concerning the MM part, the OPLS/AA force field ${ }^{60}$ has been used for the complex, and the SPC model ${ }^{61}$ has been chosen for the water molecules. The effective point charges of the water molecules were included in the quantum mechanical Hamiltonian within an electrostatic embedding scheme. More technical details on the calculations can be found in $\operatorname{Refs}^{52,57}$.

Initial conditions for the surface hopping dynamics and sampling points for calculating the vibrational broadening of UV spectra were computed in the framework of the nuclear ensemble method $^{62,63}$. For the gas phase, 150 sampling points were created from a harmonic-oscillator Wigner ${ }^{62,64}$ distribution. Ten states were computed for each of these geometries. In the case of the aqueous solution, the sampling of geometries was carried out by means of a mixed scheme applying the Wigner distribution for the QM part and performing a classical molecular dynamics (MD) to sample solvent structures. In short, the following steps were executed: (i) sample solvent structures were obtained from ground state trajectory keeping the QM solute fixed at the equilibrium structure; (ii) initial conditions were calculated for the isolated QM system from a Wigner distribution; (iii) the equilibrium QM structure in each of the solvent samples was replaced by different displaced structure from the Wigner distribution; and (iv) each structure was rethermalized for $10 \mathrm{ps}$. In this way, a sampling of the QM part with appropriate consideration of quantum mechanical zero-point energies has been achieved and combined with MD sampling for the solvent. For more details, see the discussion in $\operatorname{Refs}^{52,57}$. The initial conditions, created in that way, were also used for the calculation of the spectra using the nuclear ensemble method.

In the nonadiabatic surface hopping dynamics, the fewest switches algorithm ${ }^{48}$ corrected for decoherence effects $\left(\alpha=0.1\right.$ Hartree $\left.^{65}\right)$ was used. Nonadiabatic interactions were computed according to the numerical scheme proposed by Hammes-Schiffer and Tully ${ }^{66}$, and using the local diabatization approximation ${ }^{67,68}$. Nonadiabatic interactions between excited states were calculated employing the approximate derivative couplings orbital derivative (OD) ${ }^{69}$ method. Since the $\mathrm{ADC}(2)$ method does not describe the multireference character of the ground state well at 
intersection structures with $\mathrm{S}_{0}$, an approximate criterion has been adopted to estimate the $\mathrm{S}_{1} / \mathrm{S}_{0}$ crossings in the form of a stopping condition which is activated when the $S_{1} / S_{0}$ energy gap falls below $0.2 \mathrm{eV}$. The corresponding time step was taken as an estimate of the $\mathrm{S}_{1} / \mathrm{S}_{0}$ crossing time. It was found previously that this procedure reproduced the nonadiabatic relaxation dynamics quite well $^{70}$. The maximum simulation time was 1 ps for both gas and solution phases. The velocity Verlet algorithm ${ }^{71,72}$ with a time step of $0.5 \mathrm{fs}$ was used for the integration of Newton's equations. The Andersen-Lowe thermostat ${ }^{73}$ was applied to keep the temperature at $298 \mathrm{~K}$ in the aqueous solution. The action of the thermostat was restricted to the solvent molecules. The usual protocol to select initial conditions for dynamics considering a target diabatic character in the adiabatic state manifold is based on the difference of oscillator strengths between the states in the excitation region. Nevertheless, in the current case, such protocol did not help to select a target diabatic state due to the similarity of the oscillator strengths of the states (see Table 1). For this reason, we decided to start all trajectories from $\mathrm{S}_{4}$, which should maximize the contribution of local excitation in the initial state composition. 109 trajectories were started in each case. For the calculation of the line shapes of the spectra, the excitation at each sample point was broadened by a Lorentzian with a broadening parameter of $0.1 \mathrm{eV}$.

The value of charge transfer from fragment A to fragment B for excited states using the descriptor $q(\mathrm{CT})^{67}$ for a given electronic transition was analyzed by means of transition density matrices $\mathbf{D}^{0,[A O]}$ defining the descriptor $\Omega_{A B}^{n}$ as

$$
\Omega_{A B}^{n}=\frac{1}{2} \sum_{\substack{a \in A \\ b \in B}}\left(D^{0 n,[A O]} S^{[A O]}\right)_{a b}\left(S^{[A O]} D^{0 n,[A O]}\right)_{a b},
$$

where $n$ labels the electronic state, and $\mathbf{S}^{[\mathrm{AO}]}$ is the AO orbital overlap matrix. $\Omega_{A B}^{n}$ represents the contribution of charge transfer from fragment $\mathrm{A}$ to fragment $\mathrm{B}$ (for $\mathrm{A} \neq \mathrm{B}$ ), and the contributions of the same-fragment excitations (for $\mathrm{A}=\mathrm{B}$ ). The total CT character for a system with multiple fragments is given by:

$$
q(C T)=\frac{1}{\Omega^{n}} \sum_{i} \sum_{B \neq A} \Omega_{A B}^{n},
$$

$\Omega^{n}$ is the total sum of the charge transfer numbers for all pairs of $\mathrm{A}$ and $\mathrm{B}$. If $q(\mathrm{CT})=1 e$, a complete charge transfer of one electron has occurred while for $q(\mathrm{CT})=0$ the transition is a locally excited or Frenkel excitonic state. 
The nonadiabatic dynamics calculations were performed with the NEwTON-X program system $^{74}$. The QM calculations were all performed with TURBOMOLE ${ }^{75}$; for the MM calculations, TINKER $^{76}$ was used. Natural Transition orbitals (NTOs) ${ }^{77}$ and values $q(\mathrm{CT})$ for the charge transfer from anthracene to TCNE in excited states have been calculated using the TheoDORE ${ }^{67,78,79}$ program.

\section{Results and Discussions}

\subsection{The UV spectrum}

Vertical excitation energies, oscillator strengths, and CT values $\mathrm{q}(\mathrm{CT})$ are collected in Table 1 for the five lowest excited singlet states of the anthracene-TCNE complex for the gas phase and for continuum solvation. Table S1 of the Supporting Information (SI) shows excitation energies for all 10 states calculated in the gas phase, and Table S2 gives the corresponding results for continuum solvation. In Tables S3 and S4, the vertical excitations of the monomers anthracene and TCNE, respectively, are collected for comparison.

In the gas phase, the first two excited states of the anthracene-TCNE complex (Table 1) show a strong CT character with almost a full charge transferred to the TCNE. The LE states follow as states $\mathrm{S}_{3}$ and $\mathrm{S}_{4}$. They are localized on anthracene, while $\mathrm{S}_{5}$ and $\mathrm{S}_{6}$ have $\mathrm{CT}$ character again (Figure 2). The excitation energies of the two LE states on anthracene agree well with the ones for the isolated anthracene shown in Table S3. Excitations in TCNE start at $5.37 \mathrm{eV}$ for the isolated compound (Table S4). The first state with significant TCNE character appears in the complex as $\mathrm{S}_{9}(5.55 \mathrm{eV})$. In water (Table 1), the first three excited states have a strong CT character. $\mathrm{S}_{4}$ and $\mathrm{S}_{5}$ are locally excited states on anthracene, and $\mathrm{S}_{6}$ has $\mathrm{CT}$ character. The effect of the solvent is to stabilize the CT states by $0.3-0.5 \mathrm{eV}$, which brings the CT(III) state to the third excited state. The LE states are practically unaffected by the polar solvation. This situation in terms of state ordering and character of states is in quite good agreement with the results obtained in Ref. ${ }^{27}$ using a much larger cc-pVTZ basis. Differences in terms of excitation energies amount to a few tenths of an $\mathrm{eV}$. Comparison of the calculated results for aqueous solution with spectroscopic data for dichloromethane given in Table 1 shows a good representation of the first two CT bands found in the experiments. Comparison of available spectroscopic data for anthracene (Table S3) and TCNE (Table S4) demonstrate also good agreement for our calculated results. 
Table 1. Vertical excitation energies $\Delta \mathrm{E}(\mathrm{eV})$, oscillator strengths $\mathrm{f}$, charge transfer $\mathrm{q}(\mathrm{CT})$ in $e$, and most important orbital transitions computed for the TCNE/anthracene complex at the ground state minimum using the SOS-ADC(2)/SV(P) method.

\begin{tabular}{|c|c|c|c|c|}
\hline State & $\Delta E$ & $f$ & $q(\mathrm{CT})$ & Character $^{\mathrm{a}}$ \\
\hline \multicolumn{5}{|c|}{ Gas phase } \\
\hline $\mathrm{S}_{1}(\mathrm{CT}(\mathrm{I}))$ & $\begin{array}{c}2.38 \\
(2.22)^{\mathrm{b}}\end{array}$ & 0.15 & 0.89 & $\mathrm{H} \rightarrow \mathrm{L}(99 \%)$ \\
\hline $\mathrm{S}_{2}(\mathrm{CT}(\mathrm{II}))$ & $\begin{array}{c}3.40 \\
(3.24)^{b}\end{array}$ & 0.00 & 0.93 & $\mathrm{H}-1 \rightarrow \mathrm{L}(99 \%)$ \\
\hline $\mathrm{S}_{3}(\mathrm{LF}$ (I)) & 3.85 & 000 & 026 & $\mathrm{H}-1 \rightarrow \mathrm{L}+1(43 \%)$ \\
\hline $\mathrm{S}_{3}(\mathrm{LE}(1))$ & $(3.78)^{b}$ & & & $\mathrm{H}-1 \rightarrow \mathrm{L}+2(30 \%)$ \\
\hline $\mathrm{S}_{4}(\mathrm{LE}(\mathrm{II}))$ & 3.96 & 0.05 & 0.07 & $\mathrm{H} \rightarrow \mathrm{L}+1(92 \%)$ \\
\hline $\mathrm{S}_{5}(\mathrm{CT}(\mathrm{III}))$ & 4.01 & 0.02 & 0.71 & $\mathrm{H}-2 \rightarrow \mathrm{L}(74 \%)$ \\
\hline $\mathrm{S}_{6}(\mathrm{CT}(\mathrm{IV}))$ & 4.98 & 0.02 & 0.89 & $\mathrm{H}-4 \rightarrow \mathrm{L}(96 \%)$ \\
\hline \multicolumn{5}{|c|}{ Water (COSMO) } \\
\hline $\mathrm{S}_{1}(\mathrm{CT}(\mathrm{I}))$ & $\begin{array}{c}1.98 \\
(1.71)^{\mathrm{c}}\end{array}$ & 0.19 & 0.86 & $\mathrm{H} \rightarrow \mathrm{L}(98 \%)$ \\
\hline $\mathrm{S}_{2}(\mathrm{CT}(\mathrm{II}))$ & $\begin{array}{c}2.97 \\
(2.75)^{\mathrm{c}}\end{array}$ & 0.00 & 0.91 & $\mathrm{H}-1 \rightarrow \mathrm{L}(99 \%)$ \\
\hline $\mathrm{S}_{3}(\mathrm{CT}(\mathrm{III}))$ & $\begin{array}{c}3.56 \\
(3.34)^{\mathrm{c}}\end{array}$ & 0.03 & 0.75 & $\mathrm{H}-2 \rightarrow \mathrm{L}(80 \%)$ \\
\hline $\mathrm{S}_{4}(\mathrm{LE}(\mathrm{I}))$ & 3.90 & 0.06 & 0.12 & $\mathrm{H} \rightarrow \mathrm{L}+1(92 \%)$ \\
\hline $\mathrm{S}_{5}(\mathrm{LE}(\mathrm{II}))$ & 3.94 & 0.02 & 0.22 & $\begin{array}{l}\mathrm{H} \rightarrow \mathrm{L}+2(40 \%) \\
\mathrm{H}-1 \rightarrow \mathrm{L}+1(39 \%)\end{array}$ \\
\hline $\mathrm{S}_{6}(\mathrm{CT}(\mathrm{IV}))$ & 4.55 & 0.03 & 0.87 & $\mathrm{H}-4 \rightarrow \mathrm{L}(96 \%)$ \\
\hline \multicolumn{5}{|l|}{ Exp. } \\
\hline CT band I & 1.73 & & & \\
\hline CT band II & 2.79 & & & \\
\hline
\end{tabular}


${ }^{a} \mathrm{H}$ stands for HOMO and L for LUMO; the percentage numbers in parentheses give the weight of the configuration in the linear-response expansion. ${ }^{b} \mathrm{cc}-\mathrm{pVTZ}$ results from Ref. ${ }^{27} .{ }^{\mathrm{c}} \mathrm{cc}-$ pVTZ results from Ref. ${ }^{27}$ for dichloromethane. ${ }^{\mathrm{d}}$ Ref. ${ }^{26}$, for dichloromethane solution.

The orbital excitation scheme is illustrated in Figure 2, showing the lowest excited states generated from the list of molecular orbitals (MO) ranging from highest occupied MO (HOMO) to HOMO-2 and the lowest unoccupied MO (LUMO) to LUMO+2.

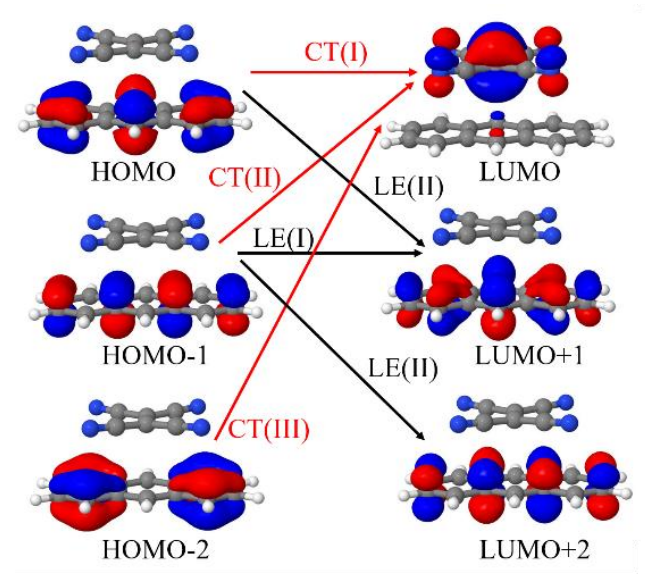

Figure 2. MO scheme and the characterization of the vertical transitions for the anthracene-TCNE complex in gas phase; red lines indicate CT transitions, and black lines refer to localized excitation in anthracene.

The optimized structures for the ground state and first excited state in the gas phase using the SOS-MP2/SV(P) and SOS-ADC (2)/SV(P) methods, respectively, are displayed in Figure 3 together with bond and intermolecular distances. The Cartesian coordinates are collected in the SI, Table S6. The intermolecular distance increases from $3.14 \AA$ in the ground state to $3.25 \AA$ in $\mathrm{S}_{1}$. The change in the $\mathrm{C}=\mathrm{C}$ bond distance from $1.38 \AA$ in $\mathrm{S}_{0}$ to $1.46 \AA$ in $\mathrm{S}_{1}$ due to $\mathrm{CT}$ populating the antibonding $\pi^{*}$ orbital is most prominent. Another characteristic change due to CT in $\mathrm{S}_{1}$ is a symmetric torsion across the $\mathrm{C}=\mathrm{C}$ bond of $\mathrm{TCNE}$ with the electronegative nitrogen ends pointing more to anthracene. 

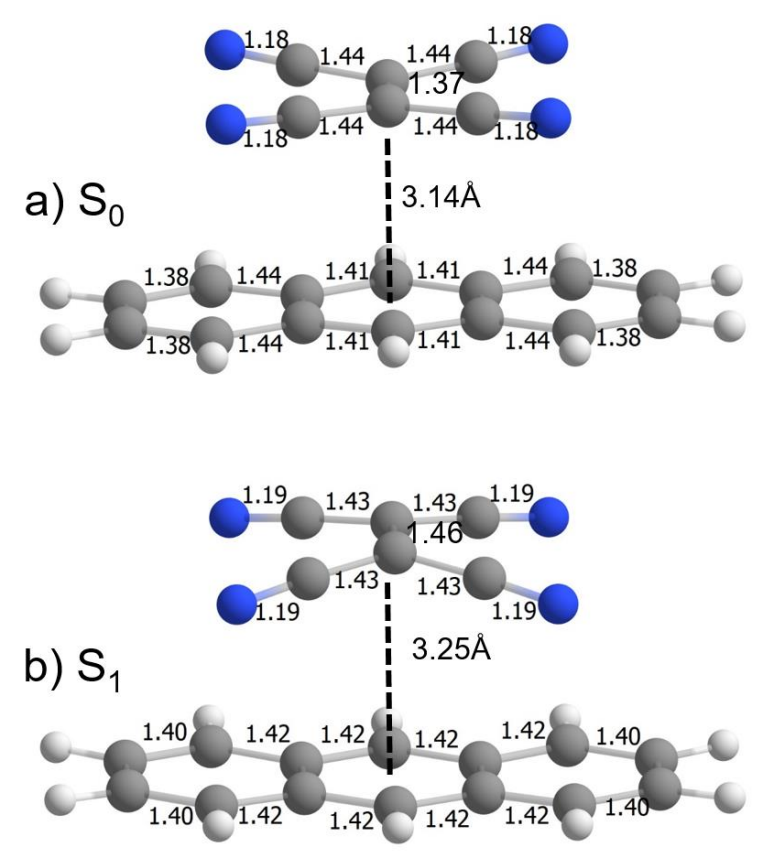

Figure 3. Bond distances $(\AA)$ and center-of-mass distances $(\AA)$ (dashed line) for (a) the $\mathrm{S}_{0}$ state optimized at SOS-MP2/SV(P) level and (b) the $\mathrm{S}_{1}$ structure optimized at SOS-ADC(2)/SV(P) level in the gas phase. The trans-CCCC torsional angle in TCNE is $175^{\circ}$ in $\mathrm{S} 0$ and $166^{\circ}$ in $\mathrm{S}_{1}$.

The nuclear ensemble spectrum of the TCNE/anthracene complex in the isolated and solution phases is shown in Figure 4. There are two absorption bands in the region of up to $4 \mathrm{eV}$. The first band originates from CT transitions. LE and CT transitions contribute to the second band system. At even higher energies, an intensive band consisting of LE transitions, which is partially shown in Figure 4. In the case of the gas phase, the band maxima are found at $2.19 \mathrm{eV}$ and 3.80 $\mathrm{eV}$ while they are located at $\sim 1.68 \mathrm{eV}$ and $3.72 \mathrm{eV}$ in solution. The redshift induced by solvation is $0.51 \mathrm{eV}$ for the first (CT) peak while it is much less $(0.08 \mathrm{eV})$ for the second peak. The positions and relative intensities of the vertical emissions for solution are given in Figure 4 as well. See Tables S1 and S2 for numeric values for gas phase and solution. 


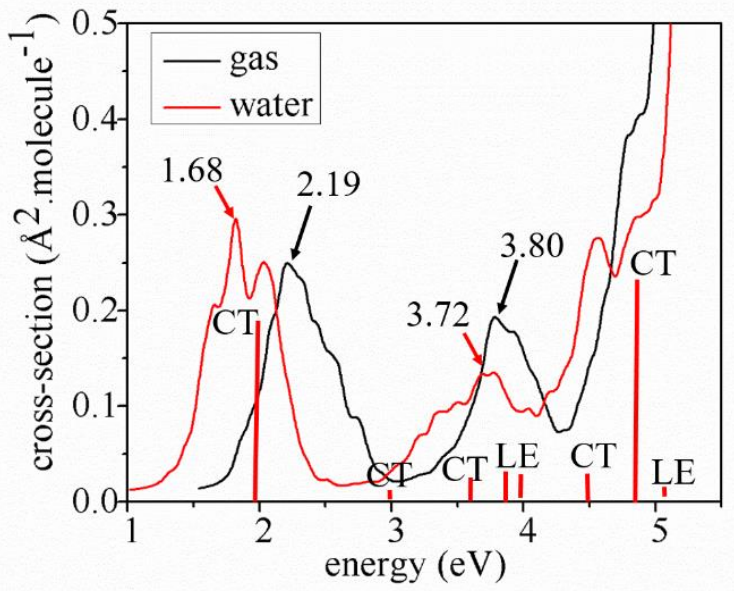

Figure 4: Photoabsorption cross-section in $\AA^{2}$.molecule ${ }^{-1}$ as a function of the excitation energy $(\mathrm{eV}) ; 150$ points and 10 states were calculated for both gas and aqueous solution. Red vertical lines (arbitrary units) show the excitation energies for each transition and their character in solution.

The vertical emission energy ( $\left.\Delta \mathrm{E}_{\text {emission}}\right)$ from the geometry optimized in the $\mathrm{S}_{1}$ state to $\mathrm{S}_{0}$ is compared in Table 2 with the vertical absorption $\left(\Delta \mathrm{E}_{\text {vert }}\right)$ and the adiabatic $\left(\Delta \mathrm{E}_{\text {adiab }}\right) \mathrm{S}_{0} / \mathrm{S}_{1}$ excitation energy. The latter is computed as the difference between the $\mathrm{S}_{0}$ and $\mathrm{S}_{1}$ energies computed at the respective optimized geometries. In the gas phase, the geometry relaxation in $\mathrm{S}_{1}$ reduces its energy by $0.45 \mathrm{eV}$, yielding $1.44 \mathrm{eV}$ emission energy. This relaxation renders a substantial Stokes shift $\left(\Delta \mathrm{E}_{\mathrm{vert}}-\Delta \mathrm{E}_{\text {emission }}\right)$ of $0.94 \mathrm{eV}$. In water, $\Delta \mathrm{E}_{\text {emission }}$ is negative, indicating that solvation stabilizes the $\mathrm{S}_{1} \mathrm{CT}$ state considerably, but also that the description SOS-ADC(2) is breaking down since it is not capable of taking into account the multireference character of the ground state at the intersection $^{80}$, as already discussed in the Computational Details.

Table 2. Vertical absorption energy to $S_{1}\left(\Delta E_{\text {vert }}\right.$ ), adiabatic $S_{0} / S_{1}$ energy ( $\left.\Delta E_{\text {adiab }}\right)$ and vertical emission energy ( $\left.\triangle \mathrm{E}_{\text {emission }}\right)$ from $\mathrm{S}_{1}$ to $\mathrm{S}_{0}$ for the TCNE/anthracene complex in gas phase and in aqueous solution (COSMO).

\begin{tabular}{cccc} 
& $\Delta \mathrm{E}_{\text {vert }}(\mathrm{eV})$ & $\Delta \mathrm{E}_{\text {adiab }}(\mathrm{eV})$ & $\Delta \mathrm{E}_{\text {emission }}(\mathrm{eV})$ \\
\hline Gas phase & 2.38 & 1.93 & 1.44 \\
Water (COSMO) & 1.98 & $0.88^{\mathrm{a}}$ & $0.51^{\mathrm{a}, \mathrm{b}}$
\end{tabular}

${ }^{a}$ In this transition, the energy of the excited state computed with linear response is lower than that of the ground (reference) state. ${ }^{b}$ Optimized $\mathrm{S}_{1}$ geometry of the isolated complex used. 


\subsection{The Photo-dynamics of the Anthracene-TCNE Complex}

The dynamics simulations have been started in both the gas and aqueous solution phases from $\mathrm{S}_{4}$, which is predominantly a LE state. Figure 5 shows the evolution of the average state occupations over all trajectories. In the gas phase, the decay of the initial $\mathrm{S}_{4}$ state occurs within a few fs leading to a concomitant rapid initial increase of $S_{3}$, which subsequently decreases steadily until the end of the simulation time. The state occupation then switches to $\mathrm{S}_{2}$, which has a maximum at around $110 \mathrm{fs}$ and decreases to about $15 \%$ within the simulation time of 1 ps. The decrease in $\mathrm{S}_{2}$ is accompanied by a steady increase in $\mathrm{S}_{1}$. The occupation of the $\mathrm{S}_{0}$ state increases slowly due to the decay of the $S_{1}$ state.
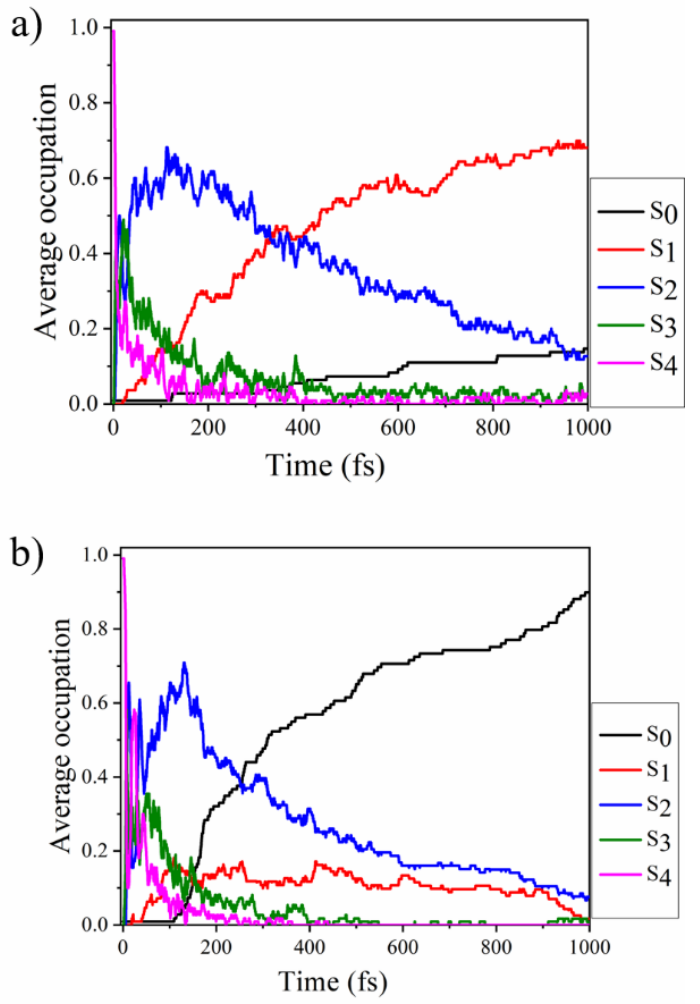

Figure 5: Average state occupation for the photo-dynamics of the anthracene-TCNE complex a) for the gas phase and $b$ ) for water.

In solution, the $\mathrm{S}_{4}$ (LE) state also decays very fast. It reaches an occupation of $<10 \%$ in less than $15 \mathrm{fs}$. Similar to the gas phase, a maximum in the $\mathrm{S}_{2}$ occupation is observed at $\sim 130 \mathrm{fs}$. In contrast to the gas phase, in solution, the $S_{1}$ state decays rapidly to $S_{0}$, which is populated at the 
end of the simulation time by $90 \%$.

The CT behavior within a trajectory is shown in Figure 6 for both gas and solution phases. Figure $6 \mathrm{a}$ and $\mathrm{b}$ show the CT values for all states calculated during the dynamics and provide a good overall picture of possible CT character. On the other hand, Figure $6 \mathrm{c}$ and $\mathrm{d}$ focus on the CT character of the active state, i.e., the state that is actually occupied at a given time in the surfacehopping dynamics. $\mathrm{S}_{4}$ has, most of the time, LE character (Figure 6a,b), both in the gas phase and solution. $\mathrm{S}_{3}$ shows stronger variations in the gas phase, but both states do not play an important role overall since the above discussion showed that they decay rapidly to lower states. From Figure 6a, a similar dynamic behavior of the CT character of $S_{1}$ and $S_{2}$ can be seen, irrespective of their actual occupation. Except for a short period in the beginning, the evolution of the CT in the active state (Figure 6c, gas phase) displays a continuous strong CT character while switching from one CT state to the other. In solution (Figure 6b), the density of CT states comprises now three states $\left(\mathrm{S}_{1}-\mathrm{S}_{3}\right)$. Figure $6 \mathrm{c}$ in gas and Figure $6 \mathrm{~d}$ in solution phases, clearly show this behavior of active state switching of CT from the $\mathrm{S}_{4}$ state to the $\mathrm{S}_{1}$ state. Figure $6 \mathrm{~d}$ shows an interesting feature of initial strong oscillations in $\mathrm{q}(\mathrm{CT})$ and switches between different states. 

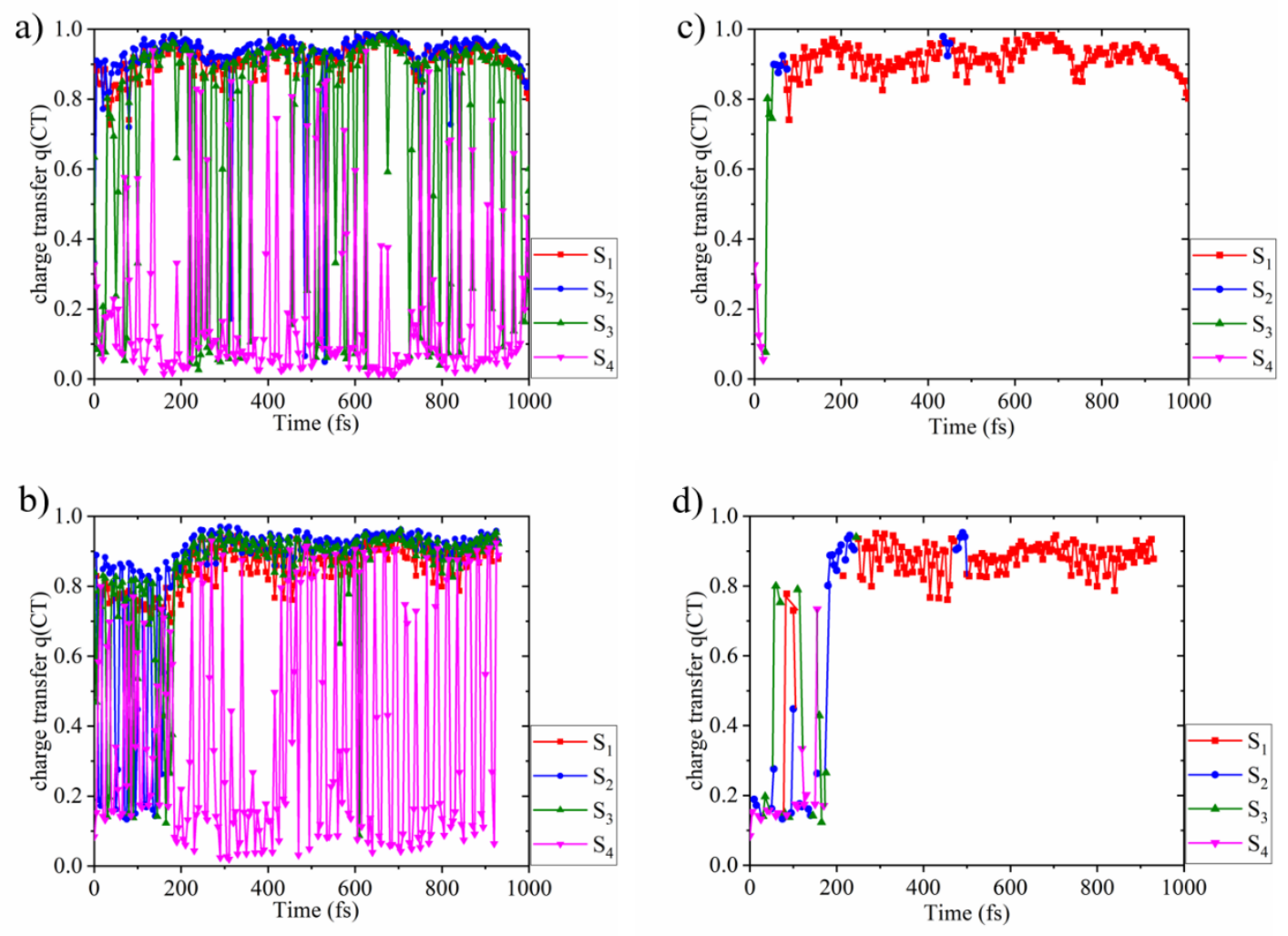

Figure 6: Charge transfer character during the photo-dynamics of anthracene-TCNE for a selected trajectory a) all states for gas phase, b) all states for solution phase, c) active state in the gas phase, d) active state in solution.

The discussion of Figure 6 just above showed that, except for an initial phase, the character of the lowest excited states is dominated by CT character. Therefore, we did not follow their decay individually but treated them globally as one CT entity. To estimate their combined excited-state lifetime, we fitted the quantity

$$
f_{e}(t)=1-f_{0}(t),
$$

where $f_{0}(t)$ is the occupation of $\mathrm{S}_{0}$, with the exponential decay function

$$
N_{0}(t)=N_{1}+\left(1-N_{1}\right) e^{-\left(t-t_{L}\right) t_{e}} .
$$

In this equation, $N_{1}$ is the fraction of the population that does not decay to the ground state in the ultrafast process, $t_{L}$ is the latency time until the hops to $\mathrm{S}_{0}$ start, and $t_{e}$ is the exponential time constant. The excited-state lifetime is given as $\tau_{10}=t_{e}+t_{L}$. Because our simulations are limited to 
$1 \mathrm{ps}$, we do not have enough information to predict the long time scale fate of the system fully. Thus, for both phases, we fitted the data twice, keeping $N_{1}$ fixed at zero (which implies the hypothesis that the entire excited state population goes to the ground state through the same ultrafast process) and optimizing it (which leaves open the possibility that part of the excited state population decays through another, longer process).

We have also estimated the time $\tau_{32}$ to transfer the population from the high excited states $S_{4}$ and $S_{3}$ to $S_{2}$ and the time $\tau_{21}$ to transfer the population from $S_{2}$ and $S_{1}$. These times were obtained by fitting the $\mathrm{S}_{2}$ occupation with the unimolecular decay function

$$
N_{2}(t)=\frac{\tau_{21}}{\tau_{21}-\tau_{32}}\left[e^{-t / \tau_{21}}-e^{-t / \tau_{32}}\right] .
$$

Table 3. Time constants derived from fitting the gas phase and solution dynamics, according to Eqs. (5) and (6).

\begin{tabular}{lcccccc}
\hline & $N_{1}$ & $t_{L}(\mathrm{fs})$ & $t_{e}(\mathrm{fs})$ & $\tau_{10}(\mathrm{fs})$ & $\tau_{21}(\mathrm{fs})$ & $\tau_{32}(\mathrm{fs})$ \\
\hline Gas phase $\left(N_{1}\right.$ free $)$ & 0.59 & 122 & 1985 & 2107 & 395 & 73 \\
Gas phase $\left(N_{1}\right.$ fixed $)$ & 0 & 122 & 5316 & 5438 & & \\
Water $\left(N_{1}\right.$ free $)$ & 0.08 & 110 & 341 & 451 & 266 & 69 \\
Water $\left(N_{1}\right.$ fixed $)$ & 0 & 110 & 404 & 514 & & \\
\hline
\end{tabular}

In both gas and water phases, the $S_{2}$ state is populated within $100 \mathrm{fs}$, as indicated by $\tau_{32}$ in Table 3. This time marks the birth of the CT state. In the gas phase, it takes about 395 fs for the $\mathrm{S}_{2}$ state to transfer the population to $S_{1}$. In water, however, this process is faster, taking $266 \mathrm{fs}$.

In both water and the gas phases, the latency time before the first transition to the ground state is about $110-120 \mathrm{fs}$ (Table 3 ). In the gas phase, if $N_{1}$ is optimized, it yields the value $N_{1}=$ 0.59 , indicating that a substantial amount of population may remain excited and does not follow the ultrafast decay mechanism. In this case, the ultrafast excited-state lifetime $\tau$ is $2.1 \mathrm{ps}$. Forcing $100 \%$ decay to the ground state ( $N_{1}$ fixed at 0 ) enhances the lifetime considerably to $5.4 \mathrm{ps}$. In water, the situation is completely different. Either optimizing of fixing $N_{1}$, the results are similar. If $N_{1}$ is optimized, one finds that only about $8 \%$ of the population may remain in the excited state. In such a case, the excited state lifetime is $0.45 \mathrm{ps}$. Restricting $N_{1}$ to zero produces only a tiny 
enhancement of the lifetime, to $0.51 \mathrm{ps}$. In water, the margin of error for the $\tau_{10}$ lifetime is $\pm 46 \mathrm{fs}$ for a $90 \%$ confidence interval. In the gas phase, this margin of error is \pm 113 fs for the same confidence interval.

Analysis of the structural modifications due to excitation to the $S_{1}$ minimum (Figure 3) indicated that with an extension by $0.09 \AA$, the $\mathrm{C}=\mathrm{C}$ stretching in TCNE is one of the most prominent coordinates subject to changes. Figure 7 displays the mean of the $\mathrm{C}=\mathrm{C}$ distance taken over all trajectories for each time step and shows the range of the amplitudes in the form of the standard deviation. The dynamics starts off with a mean value close to the $1.37 \AA$ obtained for the $\mathrm{C}=\mathrm{C}$ distance at the ground state minimum of the complex (Figure 3), both for the gas phase and for aqueous solution. The distance increases rapidly after electronic excitation and oscillates around $\sim 1.47 \AA$, a value close to the $S_{1}$ minimum value of $1.46 \AA$. The standard deviation amounts to $\pm 0.08 \AA$ in the gas phase and to a slightly smaller value of $0.06 \AA$ in solution. The oscillations appear more pronounced in water, especially toward the end of the simulation time, possibly also due to the statistical uncertainty caused by the smaller number of surviving trajectories.
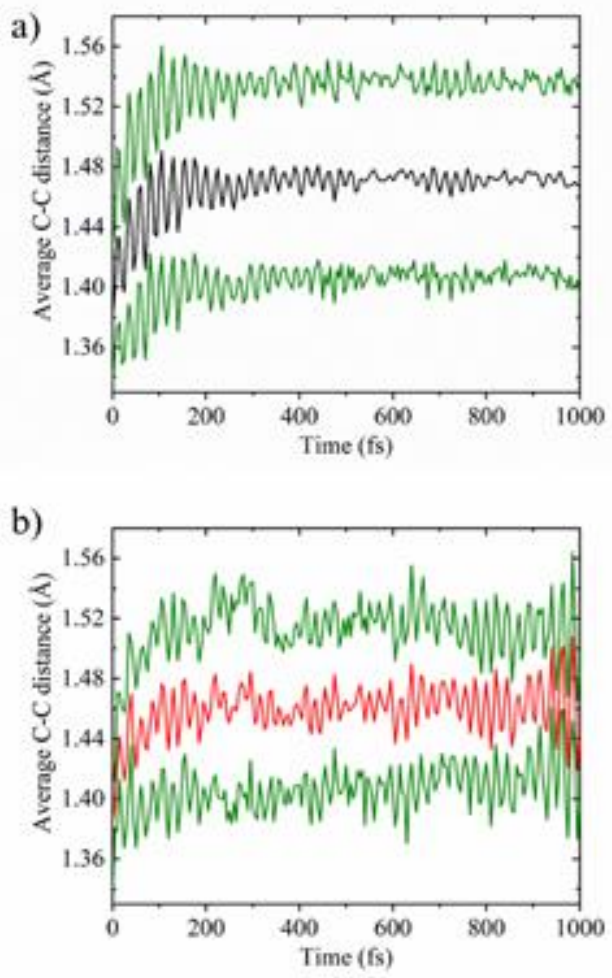
Figure 7. Time evolution of the averaged distance $(\AA)$ of a) the $\mathrm{C}=\mathrm{C}$ bond in TCNE in the gas phase (black line) and $\mathrm{b}$ ) of the $\mathrm{C}=\mathrm{C}$ bond in TCNE in water (red line). The green lines show the standard deviation around the mean value.

The center-of-mass (c.m.) distance is another interesting indicator for the description of the excited-state dynamics since it increases significantly from $3.14 \AA$ in the $\mathrm{S}_{0}$ minimum to $3.25 \AA$ in the $\mathrm{S}_{1}$ minimum (Figure 3 ). It should be mentioned that comparable increases in the intermolecular bond distances on CT were observed for several other D/A complexes as well. ${ }^{22}$ The evolution of the c.m. distances between TCNE and anthracene are shown in Figure 8. The mean c.m. distance in gas starts at $3.15 \AA$ and increases steadily in a slight oscillatory motion and reaches a limiting value of $\sim 3.3 \AA$, somewhat smaller than the $\mathrm{S}_{1}$ minimum values of $3.25 \AA$. In solution, the c.m. distances rise to a higher initial maximum at $\sim 200 \mathrm{fs}$. This increase is followed by strongly damped oscillations.
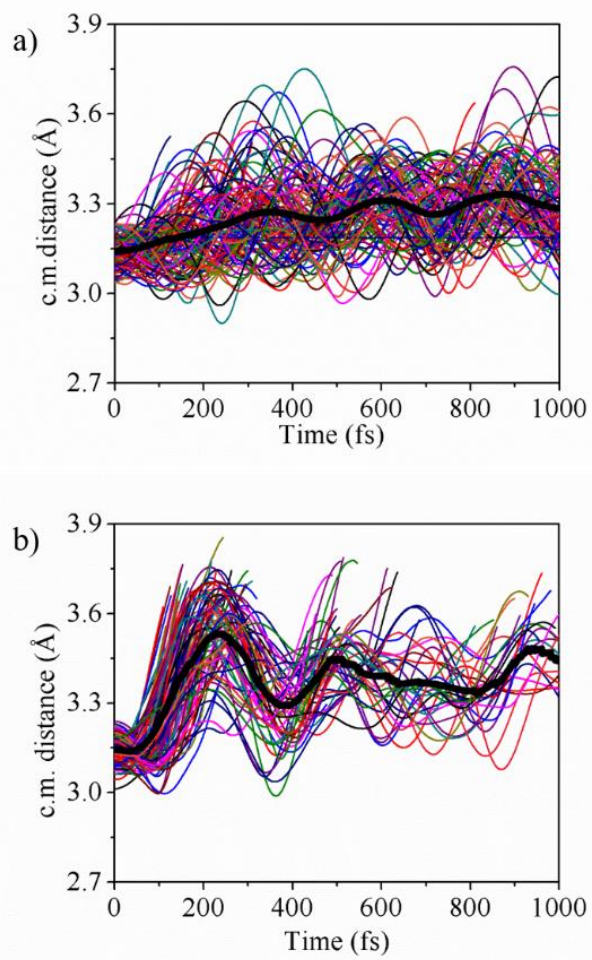

Figure 8: Center-of-mass distances between TCNE and anthracene for all trajectories as a function of simulation time. The black line shows the average distance taken over all trajectories. a) gas phase and b) in solution. 
Figure 8 also gives indications of the role the c.m. distance is playing concerning the hopping mechanism to the ground state. Inspecting this figure, one notes that, especially in the water case, the crossing between $S_{1}$ and $S_{0}$ (within our threshold criterion of $0.2 \mathrm{eV}$ ) tends to occur for c.m. distances above the mean distance. To investigate this point systematically, histograms of the c.m. and $\mathrm{C}=\mathrm{C}$ distances, respectively, at stopping time are shown in Figure 9. For both coordinates, the same picture arises: the distances at $\mathrm{S}_{1} / \mathrm{S}_{0}$ crossing time are significantly enlarged with respect to the mean value calculated over the entire dynamics. Only a few values are located below the mean value.
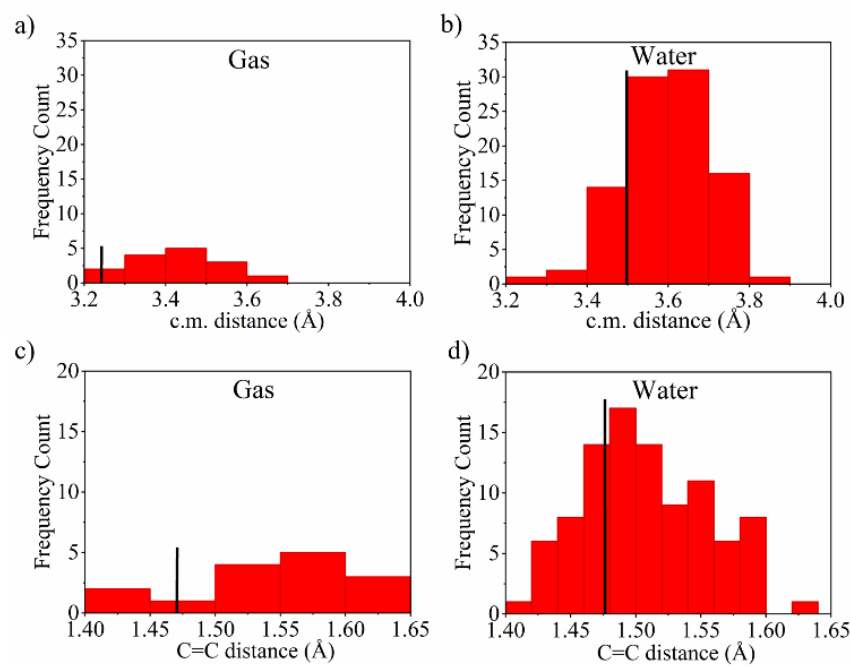

Figure 9. Histograms of the c.m. distance a) for the gas phase and b) for water, and of the $\mathrm{C}=\mathrm{C}$ distance of TCNE c) for gas phase and d) for water at the $\mathrm{S}_{1} / \mathrm{S}_{0}$ hopping time in comparison to the mean distances during the dynamics (vertical black lines).

The c.m. distance is of special interest since it will cover a larger range of geometries. In Figure 10, we plotted the potential energy profile along the rigid displacement of the c.m. coordinate. In the gas phase (Figure 10a), the $\mathrm{S}_{0}$ and $\mathrm{S}_{1}$ curves remain well separated, whereas a crossing is found in the SOS-ADC(2)-COSMO case (Figure 10b) due to the strong stabilization of the CT state by the solvent. As already mentioned, $\mathrm{ADC}(2)$ is not appropriate to describe this crossing between the states; however, outside of its neighborhood, the reference state (always denoted $\mathrm{S}_{0} / \mathrm{ClS}$ ) always has closed-shell (ClS) character and should be adequate for performing SOS-ADC(2) calculations. 
It is very interesting to note that in aqueous solution, two minima can co-exist for the ground state: the ordinary, neutral one, with a charge transfer of about $0.3 e$, and an ion-pair (IPr). The intermolecular distance in this ion pair is about $0.5 \AA$ larger than for the closed-shell dimer. Both cases have approximately the same stability. This situation resembles closely the one found previously for the interaction of para-chloranil with tetramethyl-para-phenylenediamine ${ }^{22}$, where intersections between $\mathrm{S}_{1}$ and $\mathrm{S}_{0}$ were found as well. The photoexcitation acts as a tool to populate both minima directly in an ultrafast mechanism as our simulations show. A similar mechanism has been put forward for organic charge-transfer crystals ${ }^{81}$, where laser-induced ferroelectric order was achieved by switching from the neutral to the ion-pair state.
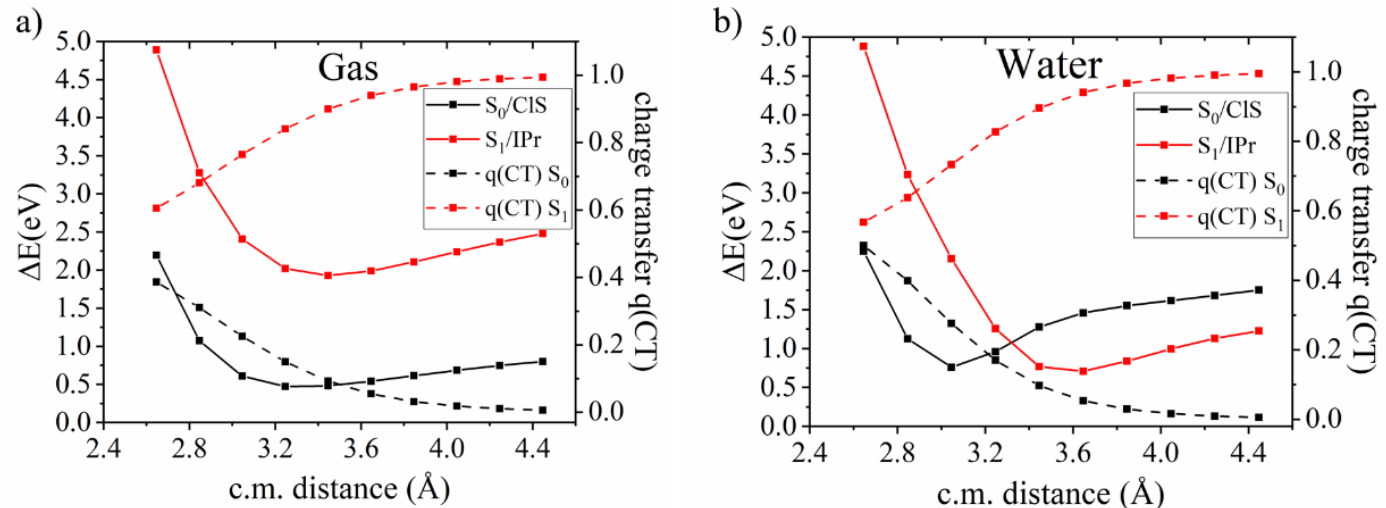

Figure 10. $\mathrm{S}_{0} / \mathrm{ClS}$ and $\mathrm{S}_{1} / \mathrm{IPr}$ potential energy curves for the rigid displacement along the line connecting the c.m. of anthracene and TCNE in the $\mathrm{S}_{1}$ optimized gas-phase structure for a) gas phase and b) water using SOS-ADC(2). Note that $\mathrm{S}_{0} / C 1 S$ and $\mathrm{S}_{1} / \mathrm{IPr}$ represent a diabatic notation with the former standing for the neutral (closed-shell) state and the latter for the ion-pair CT state. Reference energy is calculated for the optimized $\mathrm{S}_{0}$ geometry.

\section{Conclusions}

Surface hopping dynamics simulations have been performed on the benchmark system TCNE/anthracene to elucidate the photodynamical deactivation processes of LE and CT states. The process is schematically illustrated in Figure 11. The dynamics starts from the $\mathrm{S}_{4} \mathrm{LE}$ state in anthracene, which is followed by the formation of CT states within less than $100 \mathrm{fs}$. From that time on, CT states involving the transfer of almost an entire electron dominate the dynamics. The $\mathrm{S}_{2} \mathrm{CT}$ 
state reaches the maximum occupation within $\sim 110-130$ fs and depopulates towards $\mathrm{S}_{1}$, also a CT state, within 0.3 - 0.4 ps. In $\mathrm{S}_{1}$, the gas phase dynamics shows that radiationless decay may take several picoseconds. In water, however, the deactivation to $\mathrm{S}_{0}$ happens within only $0.5 \mathrm{ps}$. The most prominent geometrical changes due to dynamics in the $\mathrm{S}_{1} \mathrm{CT}$ state are the extension of the intermolecular TCNE-anthracene c.m. distance by $0.4 \AA$ and the elongation of the $\mathrm{C}=\mathrm{C}$ distance in TCNE by $0.09 \AA$. TCNE gets slightly bent with the $\mathrm{N}$ atoms moving towards anthracene. In anthracene, smaller changes in the CC bonds of $0.02 \AA$ occur, which led to almost equal bond lengths in $\mathrm{S}_{1}$.

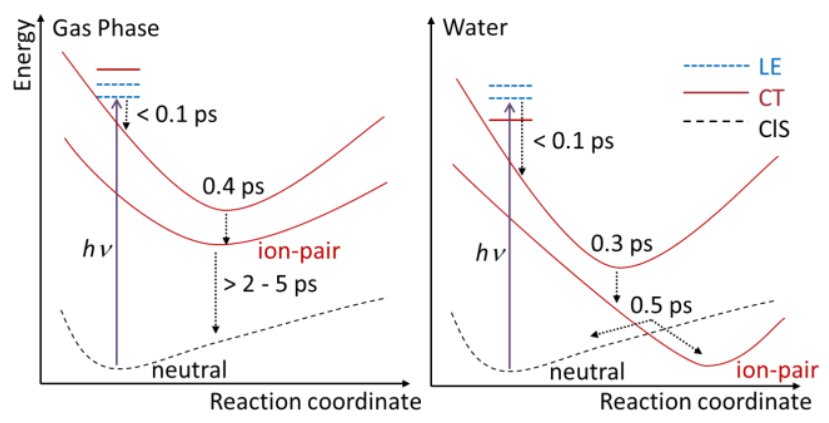

Figure 11. Schematic representation of the photodynamical process involving the LE, CT and closed-shell (ClS) states.

In the gas phase, the eventual deactivation of the complex should restore the original ground state. In water, however, a completely different picture emerges. Due to the strong stabilization of the ion-pair CT state, the ultrafast deactivation of the complex should create two different ground-state populations, one in the original neutral state and another in the ion-pair state. Although the existence of these competing ground states is clear, limitations in the computational methodology at the $\mathrm{S}_{1} / \mathrm{S}_{0}$ crossing used here preclude us from making estimates of the populations in each one.

We expect that time-resolved spectra of TCNE in the gas phase and in water should show markedly distinct features, following the scheme in Figure 11. In both environments, the spectra should feature an initial sub-picosecond time constant relative to the $\mathrm{LE} \rightarrow \mathrm{CT}$ transfer and relaxation. Nevertheless, the gas phase spectrum should give rise to a long time constant (several ps), which should not be present in the measurement in water.

The formation of a ground-state population of ion-pairs may have a profound implication for the design of photodevices. Such stable ion-pairs may have low recombination rates, which 
could increase the charge migration and separation yields. It is conceivable that such ion-pair states can also be created in solid-state, as long as the electrostatic environment is strongly polar. It is clear that the TCNE/anthracene complex is only one step toward understanding this phenomenon, and larger and more sophisticated models for the description of nonadiabatic processes are necessary.

\section{Supporting Information}

Additional Supporting Information may be found in the online version of this article concerning extended data for vertical excitations for the dimer and the monomers, and Cartesian coordiantes of optimized structures.

\section{Author Information}

Corresponding Authors

*E-mail:

adelia.aquino@univie.ac.at

hans.lischka@univie.ac.at

ORCID

Adélia J. A. Aquino: 0000-0003-4891-6512

Hans Lischka: 0000-0002-5656-3975

\section{Acknowledgments}

We are grateful for support from the School of Pharmaceutical Science and Technology (SPST), Tianjin University, Tianjin, China, including computer time on the SPST computer cluster Arran. Z. C. acknowledges financial support from the Beijing National Laboratory for Molecular Sciences (BNLMS201910). M. B. thanks the support from the projects ANR PRC WSPLIT (ANR-17CE05-0005-01) and Equip@Meso (ANR-10-EQPX-29-01). 


\section{References}

1. Mulliken, R.; Person, W. B., Donor-acceptor complexes. Annu. Rev. Phys. Chem. 1962, 13, $107-126$.

2. Metzger, R. M., The cohesive energy of tetrathiafulvalenium 7, 7, 8, 8-tetracyanoquinodimethanide (TTF TCNQ) as a function of charge transfer. J. Chem. Phys. 1981, 75, 3087-3096.

3. Roncali, J., Molecular bulk heterojunctions: an emerging approach to organic solar cells. Acc. Chem. Res. 2009, 42, 1719-1730.

4. Chen, S.; Zeng, X. C., Design of ferroelectric organic molecular crystals with ultrahigh polarization. J. Am. Chem. Soc. 2014, 136, 6428-6436.

5. $\quad$ Curutchet, C.; Mennucci, B., Quantum chemical studies of light harvesting. Chem. Rev. 2016, 117, $294-343$.

6. Hedley, G. J.; Ruseckas, A.; Samuel, I. D., Light harvesting for organic photovoltaics. Chem. Rev. 2016, 117, 796-837.

7. Valeev, E. F.; Coropceanu, V.; da Silva Filho, D. A.; Salman, S.; Brédas, J.-L., Effect of electronic polarization on charge-transport parameters in molecular organic semiconductors. J. Am. Chem. Soc. 2006, 128, 9882-9886.

8. $\quad H u$, P.; Wang, S.; Chaturvedi, A.; Wei, F.; Zhu, X.; Zhang, X.; Li, R.; Li, Y.; Jiang, H.; Long, Y., Impact of C-H... X $(X=F, N)$ and $\pi-\pi$ interactions on tuning the degree of charge transfer in F6tnap-based organic binary compound single crystals. Cryst. Growth Des. 2018, 18, 1776-1785.

9. $\quad$ Chaubey, A.; Malhotra, B., Mediated biosensors. Biosens. Bioelectron. 2002, 17, 441-456.

10. Xu, Z.; Dong, Y.; Spilling, C. D.; Lakshminarayanan, V.; Pappu, S. V. In Intermolecular charge transfer in organic donor-acceptor systems for optical storage applications, Photopolymer Device Physics, Chemistry, and Applications IV, Intern. Soc. Optics and Photonics: 1998; pp 12-18.

11. Jiang, P.; Guo, Z., Fluorescent detection of zinc in biological systems: recent development on the design of chemosensors and biosensors. Coord. Chem. Rev. 2004, 248, 205-229.

12. Tayi, A. S.; Shveyd, A. K.; Sue, A. C.-H.; Szarko, J. M.; Rolczynski, B. S.; Cao, D.; Kennedy, T. J.; Sarjeant, A. A.; Stern, C. L.; Paxton, W. F., Room-temperature ferroelectricity in supramolecular networks of charge-transfer complexes. Nature 2012, 488, 485.

13. Narayanan, A.; Cao, D.; Frazer, L.; Tayi, A. S.; Blackburn, A. K.; Sue, A. C.-H.; Ketterson, J. B.; Stoddart, J. F.; Stupp, S. I., Ferroelectric polarization and second harmonic generation in supramolecular cocrystals with two axes of charge-transfer. J. Am. Chem. Soc. 2017, 139, 9186-9191.

14. Yu, G.; Gao, J.; Hummelen, J. C.; Wudl, F.; Heeger, A. J., Polymer photovoltaic cells: enhanced efficiencies via a network of internal donor-acceptor heterojunctions. Science 1995, 270, 1789-1791.

15. Liang, Y.; Feng, D.; Wu, Y.; Tsai, S.-T.; Li, G.; Ray, C.; Yu, L., Highly efficient solar cell polymers developed via fine-tuning of structural and electronic properties. J. Am. Chem. Soc. 2009, 131, 7792-7799.

16. Thompson, B. C.; Fréchet, J. M., Polymer-fullerene composite solar cells. Angew. Chem., Int. Ed. 2008, 47, 58-77.

17. Brédas, J.-L.; Norton, J. E.; Cornil, J.; Coropceanu, V., Molecular understanding of organic solar cells: the challenges. Acc. Chem. Res. 2009, 42, 1691-1699.

18. Akimov, A. V.; Neukirch, A. J.; Prezhdo, O. V., Theoretical insights into photoinduced charge transfer and catalysis at oxide interfaces. Chem. Rev. 2013, 113, 4496-4565.

19. Stein, T.; Kronik, L.; Baer, R., Reliable prediction of charge transfer excitations in molecular complexes using time-dependent density functional theory. J. Am. Chem. Soc. 2009, 131, 2818-2820.

20. Liao, M.-S.; Lu, Y.; Parker, V. D.; Scheiner, S., DFT calculations and spectral measurements of charge-transfer complexes formed by aromatic amines and nitrogen heterocycles with tetracyanoethylene and chloranil. J. Phys. Chem. A 2003, 107, 8939-8948.

21. Borges Jr, I.; Aquino, A. J.; Köhn, A.; Nieman, R.; Hase, W. L.; Chen, L. X.; Lischka, H., Ab initio modeling of excitonic and charge-transfer states in organic semiconductors: the PTB1/PCBM low band gap system. J. Am. Chem. Soc. 2013, 135, 18252-18255.

22. Cui, Z.-h.; Aquino, A. J.; Sue, A. C.-H.; Lischka, H., Analysis of charge transfer transitions in stacked $\pi$-electron donor-acceptor complexes. Phys. Chem. Chem. Phys. 2018, 20, 26957-26967.

23. Chen, R.-x.; Aquino, A. J.; Sue, A. C.-H.; Niehaus, T. A.; Lischka, H., The characterization of charge transfer in excited states of extended clusters of $\pi$-stacked donor and acceptor complexes in lock-arm supramolecular ordering. J. Phys. Chem. A 2019. 
24. Hanazaki, I., Vapor-phase electron donor-acceptor complexes of tetracyanoethylene and of sulfur dioxide. J. Phys. Chem. 1972, 76, 1982-1989.

25. Merrifield, R.; Phillips, W., Cyanocarbon chemistry. II. 1 Spectroscopic studies of the molecular complexes of tetracyanoethylene. J. Am. Chem. Soc. 1958, 80, 2778-2782.

26. Masnovi, J.; Seddon, E.; Kochi, J., Electron transfer from anthracenes. Comparison of photoionization, charge-transfer excitation and electrochemical oxidation. Can. J. Chem. 1984, 62, 2552-2559.

27. Aquino, A. A.; Borges, I.; Nieman, R.; Köhn, A.; Lischka, H., Intermolecular interactions and charge transfer transitions in aromatic hydrocarbon-tetracyanoethylene complexes. Phys. Chem. Chem. Phys. 2014, 16, 2058620597.

28. Vydrov, O. A.; Scuseria, G. E., Assessment of a long-range corrected hybrid functional. J. Chem. Phys. 2006, 125, 234109.

29. Dreuw, A.; Weisman, J. L.; Head-Gordon, M., Long-range charge-transfer excited states in time-dependent density functional theory require non-local exchange. J. Chem. Phys. 2003, 119, 2943-2946.

30. likura, H.; Tsuneda, T.; Yanai, T.; Hirao, K., A long-range correction scheme for generalized-gradientapproximation exchange functionals. J. Chem. Phys. 2001, 115, 3540-3544.

31. Adamo, C.; Jacquemin, D., The calculations of excited-state properties with time-dependent density functional theory. Chem. Soc. Rev. 2013, 42, 845-856.

32. Laurent, A. D.; Jacquemin, D., TD-DFT benchmarks: a review. Int. J. Quantum Chem. 2013, 113, $2019-2039$.

33. Schirmer, J., Beyond the random-phase approximation: A new approximation scheme for the polarization propagator. Phys. Rev. A 1982, 26, 2395.

34. Trofimov, A.; Schirmer, J., An efficient polarization propagator approach to valence electron excitation spectra. J. Phys. B: At., Mol. Opt. Phys. 1995, 28, 2299.

35. Aquino, A. J.; Nachtigallova, D.; Hobza, P.; Truhlar, D. G.; Hättig, C.; Lischka, H., The charge-transfer states in a stacked nucleobase dimer complex: A benchmark study. J. Comput. Chem. 2011, 32, 1217-1227.

36. Panda, A. N.; Plasser, F.; Aquino, A. J.; Burghardt, I.; Lischka, H., Electronically excited states in poly (pphenylenevinylene): Vertical excitations and torsional potentials from high-level Ab initio calculations. J. Chem. Phys.A 2013, 117, 2181-2189.

37. Shi, B.; Nachtigallová, D.; Aquino, A. J.; Machado, F. B.; Lischka, H., High-level theoretical benchmark investigations of the UV-vis absorption spectra of paradigmatic polycyclic aromatic hydrocarbons as models for graphene quantum dots. J. Chem. Phys. 2019, 150, 124302.

38. Li, H.; Nieman, R.; Aquino, A. J.; Lischka, H.; Tretiak, S., Comparison of LC-TDDFT and ADC (2) methods in computations of bright and charge transfer states in stacked oligothiophenes. J. Chem. Theory Comput. 2014, 10, 3280-3289.

39. Wormit, M.; Rehn, D. R.; Harbach, P. H.; Wenzel, J.; Krauter, C. M.; Epifanovsky, E.; Dreuw, A., Investigating excited electronic states using the algebraic diagrammatic construction (ADC) approach of the polarisation propagator. Mol. Phys. 2014, 112, 774-784.

40. Hättig, C., Structure optimizations for excited states with correlated second-order methods: CC2 and ADC (2). Adv. Quantum Chem. 2005, 50, 37-60.

41. Hättig, C., Geometry optimizations with the coupled-cluster model CC2 using the resolution-of-the-identity approximation. J. Chem. Phys. 2003, 118, 7751-7761.

42. Hättig, C.; Weigend, F., CC2 excitation energy calculations on large molecules using the resolution of the identity approximation. J. Chem. Phys. 2000, 113, 5154-5161.

43. Jung, Y.; Lochan, R. C.; Dutoi, A. D.; Head-Gordon, M., Scaled opposite-spin second order Møller-Plesset correlation energy: an economical electronic structure method. J. Chem. Phys. 2004, 121, 9793-9802.

44. Hellweg, A.; Grün, S. A.; Hättig, C., Benchmarking the performance of spin-component scaled CC2 in ground and electronically excited states. Phys. Chem. Chem. Phys. 2008, 10, 4119-4127.

45. Møller, C.; Plesset, M. S., Note on an approximation treatment for many-electron systems. Phys. Rev. 1934, 46,618 .

46. Klamt, A., The COSMO and COSMO-RS solvation models. Wires Comput. Mol. Sci. 2011, 1, 699-709.

47. Lunkenheimer, B.; Köhn, A., Solvent effects on electronically excited states using the conductor-like screening model and the second-order correlated method ADC (2). J. Chem. Theory Comput. 2012, 9, $977-994$.

48. Tully, J. C., Molecular dynamics with electronic transitions. J. Chem. Phys. 1990, 93, 1061-1071.

49. Bakowies, D.; Thiel, W., Hybrid models for combined quantum mechanical and molecular mechanical 
approaches. J. Phys. Chem. 1996, 100, 10580-10594.

50. Sherwood, P., Hybrid quantum mechanics/molecular mechanics approaches. Modern Meth. and Algorithms of Quant. Chem. 2000, 3, 285-305.

51. Lin, H.; Truhlar, D. G., QM/MM: what have we learned, where are we, and where do we go from here? Theor. Chem. Acc. 2007, 117, 185.

52. Ruckenbauer, M.; Barbatti, M.; Müller, T.; Lischka, H., Nonadiabatic excited-state dynamics with hybrid ab initio quantum-mechanical/molecular-mechanical methods: solvation of the pentadieniminium cation in apolar media. J. Phys. Chem. A 2010, 114, 6757-6765.

53. Malmberg, C.; Maryott, A., Dielectric Constant of Water from 0 to 100 C. J. Res. Nat. Bureau Stand. 1956, $56,1$.

54. Eisenberg, H., Equation for the refractive index of water. J. Chem. Phys. 1965, 43, 3887-3892.

55. Cammi, R., Quantum cluster theory for the polarizable continuum model. I. The CCSD level with analytical first and second derivatives. J. Chem. Phys. 2009, 131, 164104.

56. Senn, H. M.; Thiel, W., QM/MM methods for biological systems. In Atomistic approaches in modern biology, Springer: 2006; pp 173-290.

57. Ruckenbauer, M.; Barbatti, M.; Müller, T.; Lischka, H., Nonadiabatic photodynamics of a retinal model in polar and nonpolar environment. J. Phys. Chem. A 2013, 117, 2790-2799.

58. Martínez, L.; Andrade, R.; Birgin, E. G.; Martínez, J. M., PACKMOL: a package for building initial configurations for molecular dynamics simulations. J. Comput. Chem. 2009, 30, 2157-2164.

59. D’Ans, L., Taschenbuch für Chemiker und Physiker, 3. Auflage, Band I. Springer: 1967.

60. Jorgensen, W. L.; McDonald, N. A., Development of an all-atom force field for heterocycles. Properties of liquid pyridine and diazenes. THEOCHEM 1998, 424, 145-155.

61. Berendsen, H.; Grigera, J.; Straatsma, T., The missing term in effective pair potentials. J. Phys. Chem. 1987, $91,6269-6271$.

62. Crespo-Otero, R.; Barbatti, M., Spectrum simulation and decomposition with nuclear ensemble: formal derivation and application to benzene, furan and 2-phenylfuran. Theor. Chem. Acc. 2012, 131, 1237.

63. Yamazaki, S.; Sobolewski, A. L.; Domcke, W., Molecular mechanisms of the photostability of indigo. Phys. Chem. Chem. Phys. 2011, 13, 1618-1628.

64. Barbatti, M.; Aquino, A. J.; Lischka, H., The UV absorption of nucleobases: semi-classical ab initio spectra simulations. Phys. Chem. Chem. Phys. 2010, 12, 4959-4967.

65. Granucci, G.; Persico, M., Critical appraisal of the fewest switches algorithm for surface hopping. J. Chem. Phys. 2007, 126, 134114.

66. Hammes-Schiffer, S.; Tully, J. C., Proton transfer in solution: Molecular dynamics with quantum transitions. J. Chem. Phys. 1994, 101, 4657-4667.

67. Plasser, F.; Lischka, H., Analysis of excitonic and charge transfer interactions from quantum chemical calculations. J. Chem. Theory Comput. 2012, 8, 2777-2789.

68. Granucci, G.; Persico, M.; Toniolo, A., Direct semiclassical simulation of photochemical processes with semiempirical wave functions. J. Chem. Phys. 2001, 114, 10608-10615.

69. Ryabinkin, I. G.; Nagesh, J.; Izmaylov, A. F., Fast numerical evaluation of time-derivative nonadiabatic couplings for mixed quantum-classical methods. J. Phys. Chem. Lett. 2015, 6, 4200-4203.

70. Plasser, F.; Crespo-Otero, R.; Pederzoli, M.; Pittner, J.; Lischka, H.; Barbatti, M., Surface hopping dynamics with correlated single-reference methods: $9 \mathrm{H}$-adenine as a case study. J. Chem. Theory Comput. 2014, 10, 1395-1405.

71. Verlet, L., Computer "experiments" on classical fluids. I. Thermodynamical properties of Lennard-Jones molecules. Phys. Rev. 1967, 159, 98-103.

72. Swope, W. C.; Andersen, H. C.; Berens, P. H.; Wilson, K. R., A computer simulation method for the calculation of equilibrium constants for the formation of physical clusters of molecules: Application to small water clusters. J. Chem. Phys. 1982, 76, 637-649.

73. Andersen, H. C., Molecular dynamics simulations at constant pressure and/or temperature. J. Chem. Phys. 1980, 72, 2384-2393.

74. Barbatti, M.; Ruckenbauer, M.; Plasser, F.; Pittner, J.; Granucci, G.; Persico, M.; Lischka, H., Newton-X: a surface-hopping program for nonadiabatic molecular dynamics. Wires Comput. Mol. Sci. 2014, 4, 26-33.

75. Ahlrichs, R.; Bär, M.; Häser, M.; Horn, H.; Kölmel, C., Electronic structure calculations on workstation computers: The program system turbomole. Chem. Phys. Lett. 1989, 162, 165-169. 
76. Ponder, J. W.; Richards, F. M., An efficient newton-like method for molecular mechanics energy minimization of large molecules. J. Comput. Chem. 1987, 8, 1016-1024.

77. Martin, R. L., Natural transition orbitals. J. Chem. Phys. 2003, 118, 4775-4777.

78. Plasser, F.; Wormit, M.; Dreuw, A., New tools for the systematic analysis and visualization of electronic excitations. I. Formalism. J. Chem. Phys. 2014, 141, 024106.

79. Plasser, F.; Bäppler, S. A.; Wormit, M.; Dreuw, A., New tools for the systematic analysis and visualization of electronic excitations. II. Applications. J. Chem. Phys. 2014, 141, 024107.

80. Tuna, D.; Lefrancois, D.; Wolański, Ł.; Gozem, S.; Schapiro, I.; Andruniów, T.; Dreuw, A.; Olivucci, M., Assessment of approximate coupled-cluster and algebraic-diagrammatic-construction methods for ground- and excited-state reaction paths and the conical-intersection seam of a retinal-chromophore model. J. Chem. Theory Comput. 2015, 11, 5758-5781.

81. Collet, E.; Lemée-Cailleau, M.-H.; Buron-Le Cointe, M.; Cailleau, H.; Wulff, M.; Luty, T.; Koshihara, S.-Y.; Meyer, M.; Toupet, L.; Rabiller, P., Laser-induced ferroelectric structural order in an organic charge-transfer crystal. Science 2003, 300, 612-615.

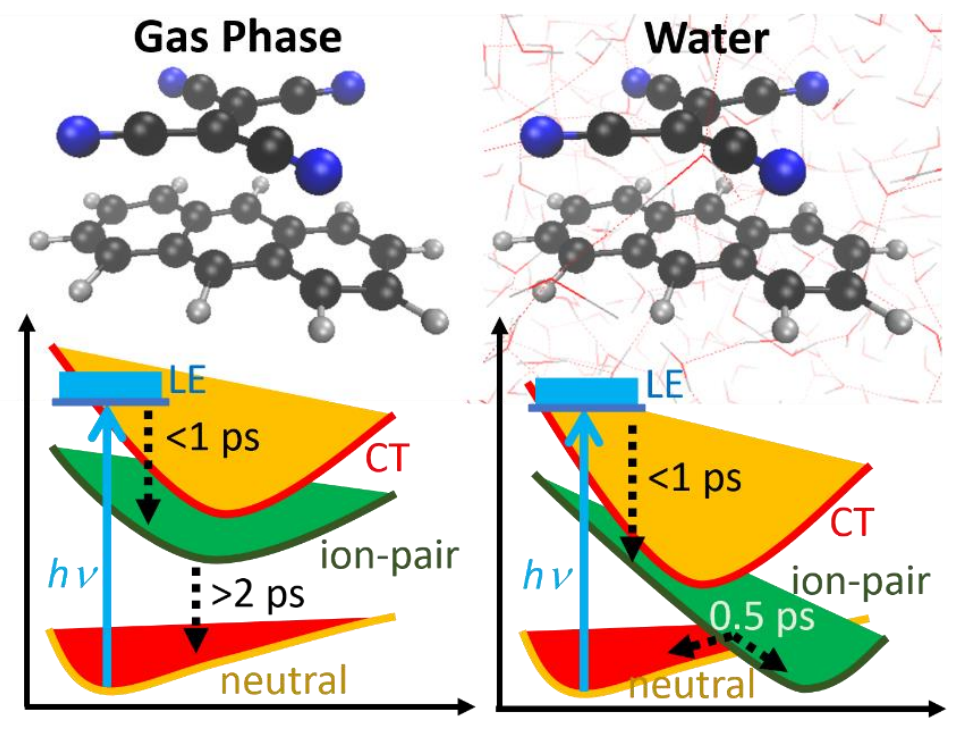

TOC Graphic 